\title{
Indirect co-culture of vascular smooth muscle cells with bone marrow mesenchymal stem cells inhibits vascular calcification and downregulates the Wnt signaling pathways
}

\author{
MENG'EN ZHU*, XIN FANG*, SHAOQIONG ZHOU, WEI LI and SIMING GUAN \\ Department of Geriatrics, Union Hospital, Huazhong University of Science and Technology, \\ Wuhan, Hubei 430022, P.R. China
}

Received March 23, 2015; Accepted December 14, 2015

DOI: $10.3892 / \mathrm{mmr} .2016 .5182$

\begin{abstract}
Vascular calcification (VC) is widely considered to be a crucial clinical indicator of cardiovascular disease. Recently, certain properties of mesenchymal stem cells (MSCs) have been hypothesized to have potential in treating cardiovascular diseases. However, their effect on the initiation and progression of $\mathrm{VC}$ remains controversial. The present study aimed to investigate whether MSCs indirectly mediate VC and their impact on the Wnt signaling pathways. A Transwell system was selected to establish the indirect co-culture environment, and hence, vascular smooth muscle cells (VSMCs) were indirectly co-cultured in the presence or absence of MSCs at a ratio of 1:1. Osteogenic medium (OS) was added to imitate a calcifying environment. Fourteen days later, VSMCs in the lower layers of the Transwell plates were harvested. Alkaline phosphatase activity and calcium nodules were markedly increased in calcific VSMCs induced by OS. However, these parameters were significantly decreased in VSMCs by indirectly co-culturing with MSCs in the same medium. Furthermore, the messenger RNA expression levels of osteopontin and osteoprotegerin were notably increased in VSMCs cultured in OS, but reduced by indirect interaction with MSCs. In addition, the activities of canonical and noncanonical Wnt ligands, wingless-type MMTV integration site family, number 5A (Wnt5a), receptor tyrosine kinase-like orphan receptor 2 (Ror2) and $\beta$-catenin, which are important in the process of $\mathrm{VC}$, were downregulated by indirect contact with MSCs in OS. Thus, indirect co-culture with MSCs inhibits VC and downregulates the Wnt signaling pathways.
\end{abstract}

Correspondence to: Professor Siming Guan, Department of Geriatrics, Union Hospital, Huazhong University of Science and Technology, 1277 Jiefang Avenue, Wuhan, Hubei 430022, P.R. China E-mail: smguan16@163.com

*Contributed equally

Key words: indirect co-culture, bone marrow mesenchymal stem cells, Wnt signaling pathways, vascular calcification

\section{Introduction}

Vascular calcification (VC) is associated with aging, hypertension, diabetes, chronic kidney diseases (CKD) and cardiovascular diseases $(1,2)$. Furthermore, it is a crucial risk factor, contributing to increased rates of cardiovascular morbidity and mortality worldwide $(1,2)$. Increasing evidence indicates that VC is an actively regulated process, similar to bone formation, including deposition of minerals in the vessel wall, and that vascular smooth muscle cells (VSMCs) undergo osteogenic differentiation with production of bone-associated biomarkers, such as osteopontin (OPN) and osteoprotegerin (OPG), are key in VC (3-6). Therefore, considering its clinical significance and complex underlying mechanisms, it is important to develop novel and efficient therapeutic strategies for preventing and inhibiting VC.

Mesenchymal stem cells (MSCs) that are extracted from bone marrow are defined as multipotent cells due to their ability to differentiate into various cell types, including VSMCs in certain conditions; however, their immunomodulatory and paracrine capabilities may present the greatest potential for therapeutic function in response to local environmental cues (7-9). Previous studies have been conducted to elucidate the two characteristics of MSCs for their application in cardiovascular disease therapy (10). Although this cell population promotes VC by differentiating into osteogenic cells in direct contact with calcified cells (11), their most notable therapeutic impacts are vascular regeneration and injury healing $(12,13)$. Additionally, MSCs promote angiogenesis via a paracrine mechanism in vivo and in vitro $(12,14)$. Therefore, it is important to analyze the anti-inflammatory, immunomodulatory and paracrine properties of MSCs on the process of $\mathrm{VC}$ using a cell-cell indirect co-culture system.

Wingless-type MMTV integration site family (Wnt) ligands encode highly conservative cysteine-rich, secreted glycoproteins and function via the activation of two intracellular signaling pathways described as canonical ( $\beta$-catenin dependent) and noncanonical ( $\beta$-catenin independent) signaling pathways (15). Activation of $\mathrm{Wnt} / \beta$-catenin signaling pathways is key in promoting VC by stimulating smooth muscle cell (SMC) differentiation into osteoblast-like cells $(15,16)$. Receptor tyrosine kinase-like orphan receptor 2 
(Ror2) is an orphan receptor tyrosine kinase that regulates osteoblastic cell proliferation and differentiation together with the $\mathrm{Wnt} / \beta$-catenin signaling pathway $(17,18)$. Additionally, noncanonical wingless-type MMTV integration site family, number 5A (Wnt5a) activates Wnt/Ror2 signaling, and enhances Wnt/ $\beta$-catenin signaling during MSC osteogenic differentiation $(19,20)$. Furthermore, a previous study indicated that increased expression of Wnt5a was correlated with VC (11). Although the cooperation among the Wnt/Ror2 and Wnt/ $\beta$-catenin signals during VC remain to be elucidated, previous studies have suggested that the Wnt signaling pathways, composed of Wnt5a and $\beta$-catenin or Ror 2 , may control the development of $\mathrm{VC}$ by regulating cell proliferation, differentiation and apoptosis.

The present study aimed to investigate whether MSCs prevent osteogenic differentiation and inhibit the development of VC in VSMCs. The effects of indirect co-culturing of MSCs with VSMCs in an OS medium on the modulation of bone-associated biomarkers and the Wnt signaling pathways were evaluated using a Transwell insert system, in vitro.

\section{Materials and methods}

Rat bone marrow MSC isolation and culture. Bone marrow MSCs were isolated from male rats (age, 3 weeks; Animal Center, Tongji Medical College, Wuhan, China) by whole marrow direct adherence (21). Rats were housed together, fed a normal diet and maintained under a light/dark cycle. The adherent cells were cultured in complete medium containing Hyclone 90\% low glucose Dulbecco's modified Eagle's medium (L-DMEM; GE Healthcare Life Sciences, Logan, UT, USA), Gibco 10\% fetal bovine serum (FBS; Thermo Fisher Scientific, Inc., Waltham, MA, USA), $100 \mathrm{U} / \mathrm{ml}$ penicillin and $100 \mathrm{U} / \mathrm{ml}$ streptomycin (Hyclone; GE Healthcare Life Sciences) in $25-\mathrm{cm}^{2}$ plastic culture flasks at $37^{\circ} \mathrm{C}$ in a $5 \% \mathrm{CO}_{2}$ supplemented incubator. The culture medium was replaced every 3-4 days. The adherent cells were treated with trypsin (GE Healthcare Life Sciences) and expanded at 80-90\% confluence. Cells at passages 3 and 4 were selected and used in the experiments. The present study was approved by the ethics committee of Huazhong University of Science and Technology (Wuhan, China) and the animals were treated according to the Guide for the Care and Use of Laboratory Animals.

Transwell co-culture system. A 12-well Transwell insert system (pore size, $0.4 \mu \mathrm{m}$; Corning Incorporated, Corning, NY, USA) was used to prevent direct contact between the cells. A-10 VSMCs (American Type Culture Collection, Manassas, VA, USA) were seeded in the lower layers of the Transwell plates at $10^{6}$ cells/well, while, MSCs were seeded in the upper layers of the Transwell plates at a ratio of 1:1. All cells were incubated at $37^{\circ} \mathrm{C}$ in $5 \% \mathrm{CO}_{2}$ with the normal experimental medium (NEM), which contained high glucose DMEM (GE Healthcare Life Sciences) supplemented with $2 \% \mathrm{FBS}, 100 \mathrm{U} / \mathrm{ml}$ penicillin and $100 \mathrm{U} / \mathrm{ml}$ streptomycin. Calcified SMCs were induced using osteogenic medium (OS), which contained NEM supplemented with $0.1 \mu \mathrm{M}$ dexamethasone (Sigma-Aldrich, St. Louis, MO, USA), $10 \mathrm{mM}$ sodium $\beta$-glycerol-phosphate (Sigma-Aldrich) and $0.05 \mathrm{mM}$ ascorbic acid-2-phosphate (Sigma-Aldrich). Cells were divided into four groups as follows: SMC group (VSMCs cultured with NEM); SMC + OS group (VSMCs cultured with OS); SMC + MSC group (co-culture group cultured with NEM); and SMC + MSC + OS group (co-culture group cultured with OS). In addition, all cells were incubated at $37^{\circ} \mathrm{C}$ in a $5 \% \mathrm{CO}_{2}$ supplemented incubator. After 14 days, cells from the four groups were harvested from the lower layers and underwent calcium quantitative testing, alkaline phosphatase (AKP) activity assay, reverse transcription-quantitative polymerase chain reaction (RT-qPCR) and western blot analysis.

Identification of MSCs. MSCs at passage 3 were collected and washed twice with phosphate-buffered saline (PBS). The cell suspensions were respectively incubated with antibodies (eBioScience, Inc., San Diego, CA, USA) against cluster of differentiation (CD)29, CD90 and CD45 in $4^{\circ} \mathrm{C}$, in the dark for $30 \mathrm{~min}$. The fluorescence intensity of the cells was measured by flow cytometry (BD Biosciences, Franklin Lakes, NJ, USA) and the results were analyzed by BD CellQuest ${ }^{\mathrm{TM}}$ Pro software (version 5.1; BD Biosciences).

Von Kossa staining. After cells in the SMC and SMC + OS groups were incubated for 14 days, VSMCs in the lower layers of the Transwell plates were used for von Kossa staining to observe the mineral deposits. The VSMCs were gently washed three times with PBS and fixed in 4\% paraformaldehyde (Wuhan Boster Biological Technology Ltd., Wuhan, China) for $30 \mathrm{~min}$ at $4^{\circ} \mathrm{C}$. Double-distilled water $\left(\mathrm{ddH}_{2} \mathrm{O}\right)$ was subsequently used for diluting the paraformaldehyde and rinsing (three times) between each step. The cells were exposed under ultraviolet light for 45 min subsequent to staining with $5 \%$ silver nitrate (Sigma-Aldrich) and immersed in 5\% sodium thiosulfate (Sigma-Aldrich) for $10 \mathrm{~min}$ to remove unreduced silver. Following rinsing, cells were stained with $1 \%$ neutral red (Sigma-Aldrich) for another $5 \mathrm{~min}$. Following washing with $\mathrm{ddH}_{2} \mathrm{O}$, the plates were dried for observation under a microscope.

AKP activity assay. VSMCs from each of the four groups were harvested in cell lysis buffer (Amresco, Solon, OH, USA) following rinsing with PBS. BCA protein assay kit (Shanghai Biyuntian Biotechnology, Co., Ltd., Shanghai, China) was used to evaluate the total cellular protein. The activity of intracellular AKP was analyzed using an AKP assay kit (Nanjing Jiancheng Bioengineering Research Institute, Nanjing, China), according to the manufacturer's protocols. All samples were measured by an automatic plate reader (Bio-Rad Laboratories, Inc., Hercules, CA, USA).

Calcium content analysis. The calcium content of each of the four groups was analyzed by calcium colorimetric assay kit (Nanjing Jiancheng Bioengineering Research Institute), which utilizes the chromogenic composite between calcium and $o$-cresolphthalein. The assay was performed following the manufacturer's protocols and all samples were analyzed by an atomic absorption spectrophotometer (Agilent Technologies, Inc., Santa Clara, CA, USA).

$R T-q P C R$. Total RNA from the above-mentioned four groups was isolated using TRIzol (Invitrogen; Thermo Fisher 
Scientific, Inc.), according to the manufacturer's protocols. The RNA was reverse transcribed into cDNA with the cDNA Syntheses kit (Invitrogen; Thermo Fisher Scientific, Inc.). RT-qPCR was conducted using an ABI PRISM 7900 sequence detector system (Applied Biosystems; Thermo Fisher Scientific, Inc.), according to the manufacturer's protocols. $\beta$-actin served as an endogenous control. PCR reactions were performed using SYBR Green/Fluorescein qPCR Master mix (2X; Thermo Fisher Scientific, Inc.), cDNA and primers. The expression level of the specific gene (the quantity of the target normalized to the endogenous control gene) was calculated using the comparative $\mathrm{Cq}$ method, $2^{-\Delta \Delta \mathrm{Cq}}$. The primer sequences for RT-qPCR were as follows: Sense, 5'-CAACAG CCGCTTCAACTCC-3' and antisense, 5'-TGACATAGC AGCACCAGTGA-3' for rat Wnt5a; sense, 5'-ACAATTTTC AGGATGACGACCA-3' and antisense, 5'-GTGATTCGG TTTTCAATCTCCC-3' for rat Ror2; sense, 5'-AACACTCAG ATGCTGTAGCCA-3' and antisense, 5'-TCTTGCTTAAAG TCATCCGTT-3' for rat $\beta$-catenin; sense, 5'-GCTGTTCTA TTCCGAATGTCT-3' and antisense, 5'-CACCAATGTCCA GTCCGAGA-3' for rat OPN; sense, 5'-ACAGTTTGCCTG GGACCAAA-3' and antisense, 5'-CGTTGCACACTGCTT TCACA-3' for rat OPG; and sense, 5'-CACGATGGAGGG GCCGGACTCATC-3' and antisense, 5'-TAAAGACCTCTA TGCCAACACAGT-3' for $\beta$-actin.

Western blot analysis. Cells from each of the four groups were lysed with a buffer comprising $50 \mathrm{mM}$ Tris, $5 \mathrm{mM}$ EDTA, $1 \mathrm{mM}$ EDT, $1 \mathrm{mM}$ ethylene glycol tetraacetic acid, $150 \mathrm{mM}$ $\mathrm{NaCl}, 1 \%$ Triton $\mathrm{X}-100,25 \mathrm{mM}$ sodium pyrophosphate, $1 \mathrm{mM}$ $\mathrm{NaF}, 1 \mathrm{mM} \beta$-glycerophosphate, $0.1 \mathrm{mM}$ sodium orthovanadate, $1 \mathrm{mM}$ phenylmethanesulfonyl fluoride, $2 \mu \mathrm{g} / \mathrm{ml}$ leupeptin and $10 \mu \mathrm{g} / \mathrm{ml}$ aprotinin (Amresco) for $20 \mathrm{~min}$ on ice. Total protein contents were measured with a BCA assay kit according to the manufacturer's protocols. The protein lysate was separated on SDS-PAGE gels (Amresco) and electroblotted onto polyvinylidene difluoride membranes (EMD Millipore, Billerica, MA, USA). Following blocking with 5\% non-fat dried milk in Tris-buffered saline (TBS) and TBS and Tween-20 (TBST) buffer, the membrane was incubated overnight at $4^{\circ} \mathrm{C}$ with primary antibodies as follows: Rabbit polyclonal anti-Wnt5a (1:10,000; cat. no. ab72583; Abcam, Cambridge, MA, USA), rabbit polyclonal anti-Ror2 (1:500, cat. no. YT4165; ImmunoWay Biotechnology, Co., Newark, DE, USA), rabbit polyclonal anti- $\beta$-catenin $(1: 1,000$; cat. no. 9562; Cell Signaling Technology, Inc., Danvers, MA, USA) and mouse polyclonal anti- $\beta$-actin (cat. no. KM9001; 1:8,000; Sanjian, Tianjin, China) The membrane was washed four times with TBST, and further incubated with horseradish peroxidase (HRP)-conjugated goat anti-mouse (cat. no. ab20043; 1:10,000; Abcam) and goat anti-rabbit (cat. no. ab97051; 1:5,000; Abcam) secondary antibodies. Following four washes with TBST, the immunoblots were visualized using the chemiluminescence detection system (Bio-Rad Laboratories, Inc.). The protein loading was normalized by re-incubating the same membrane with anti- $\beta$-actin (cat. no. KM9001; Sanjian, Tianjin, China) at a dilution of $1: 10,000$.

Statistical analysis. Data were analyzed using SPSS software (version 17.0; SPSS, Inc., Chicago, IL, USA). Results are presented as the mean \pm standard error of the mean and $\mathrm{P}<0.05$ was considered to indicate a statistically significant difference.

\section{Results}

MSC characteristics. MSCs at passage 3 were analyzed by flow cytometry. The results indicate that the MSCs were positive for CD29 (97.9\%) and CD90 (98.71\%), and negative for CD45 (7.16\%); thus these cells demonstrated expression levels characteristic of MSCs (Fig. 1).

Von Kossa staining for VSMCs. Von Kossa staining was used to evaluate whether VSMCs transformed into osteogenic cells (Fig. 2). Black calcified nodules were observed microscopically in the VSMCs cultured with OS. However, no mineral deposits were observed in the control group incubated with NEM (Fig. 2). This indicated that the OS induced VSMCs to differentiate into the osteogenic phenotype.

AKP activity and calcium content of VSMCs. The AKP activity and calcium content in VSMCs were determined to estimate the progression of calcification. The results demonstrate that following culture for 14 days, AKP activity and calcium content were significantly increased in the $\mathrm{SMC}+\mathrm{OS}$ and $\mathrm{SMC}+\mathrm{MSC}+\mathrm{OS}$ groups compared with the SMC group $(\mathrm{P}<0.001)$, and reached the highest levels in the SMC + OS group $(\mathrm{P}<0.001)$. Furthermore, the expression levels of osteogenic markers were significantly decreased in the SMC + MSC + OS group compared with the SMC + OS group $(\mathrm{P}<0.001)$. No change in AKP activity or calcium content was observed between cells in the SMC and the SMC + MSC groups (Fig. 3A and B).

Indirect contact between SMCs and MSCs reduces the $m R N A$ levels of $O P G$ and $O P N$. mRNA expression levels of OPG and OPN were evaluated and negative expression was observed in the normal VSMCs by RT-qPCR $(3,4)$. Expression levels were significantly increased in the SMC + OS group compared with the SMC group $(\mathrm{P}<0.01)$, indicating that the VSMCs have a potential to trans-differentiate into osteoblast-like cells in the presence of OS. Furthermore, compared with the $\mathrm{SMC}+\mathrm{OS}$ group, the expression levels of OPG and OPN were significantly reduced in the $\mathrm{SMC}+\mathrm{MSC}+\mathrm{OS}$ group ( $\mathrm{P}<0.01$; Fig. 4).

Wht signaling pathways are downregulated when SMCs indirectly interacted with MSCs. The mRNA and protein expression levels of Wnt5a, Ror 2 and $\beta$-catenin, which are known to modulate osteogenic differentiation, were evaluated by RT-qPCR and western blot analysis. Comprehensive analysis of the results indicated that compared with the SMC group, Wnt5a, Ror 2 and $\beta$-catenin expression levels were significantly upregulated in the $\mathrm{SMC}+\mathrm{OS}$ group $(\mathrm{P}<0.001)$, demonstrating that the Wnt signals were activated by OS. Furthermore, the expression levels of Wnt5a, Ror2 and $\beta$-catenin were downregulated in the $\mathrm{SMC}+\mathrm{MSC}+\mathrm{OS}$ group compared with the SMC + OS groups $(\mathrm{P}<0.001)$, indicating that the Wnt signaling pathways were downregulated when direct interaction with MSCs induced VSMC osteogenic differentiation (Fig. 5). 

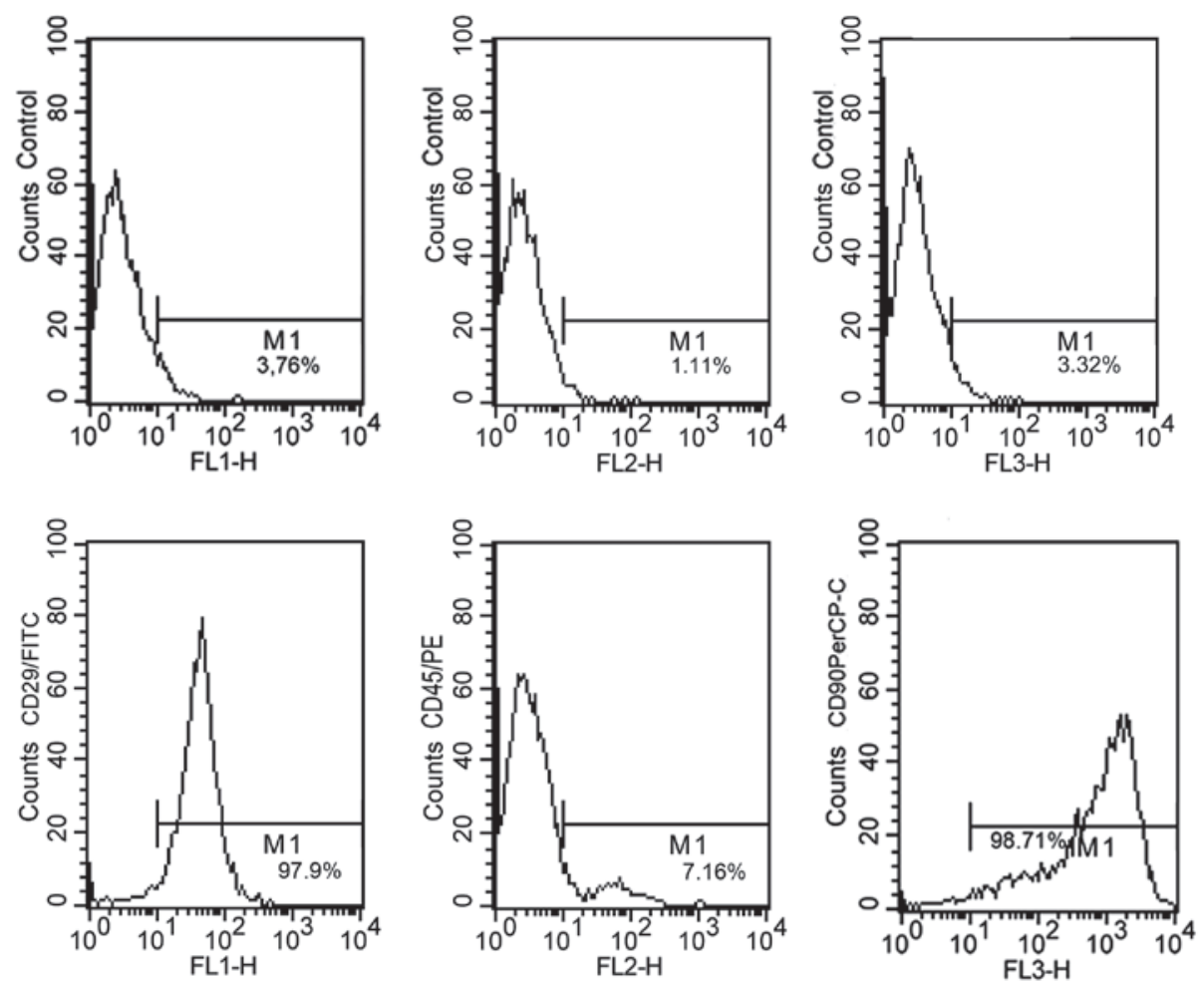

Figure 1. Mesenchymal stem cell characteristics. The expression of CD29 and CD90 was positive at 97.9 and $98.71 \%$, respectively. The expression of CD45 was negative at $7.16 \%$. CD, cluster of differentiation; FITC, fluorescein isothiocyanate.

A

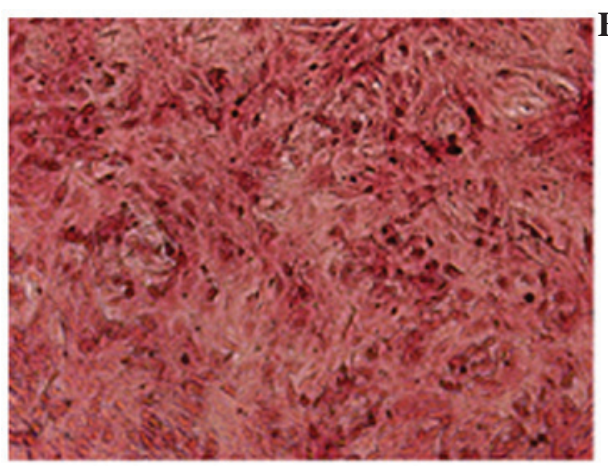

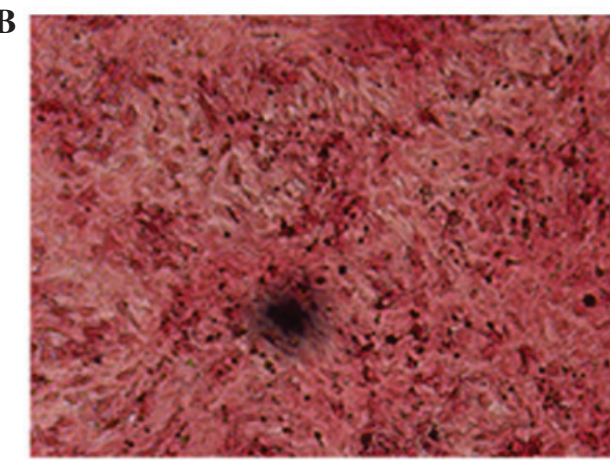

Figure 2. Von Kossa staining of VSMCs. VSMCs were cultured with or without OS for 14 days. Representative images demonstrate (A) no mineral deposits in the medium without OS and (B) black calcified nodules in the medium with OS. Magnification, x100. VSMC, vascular smooth muscle cell; OS, osteogenic medium.

A

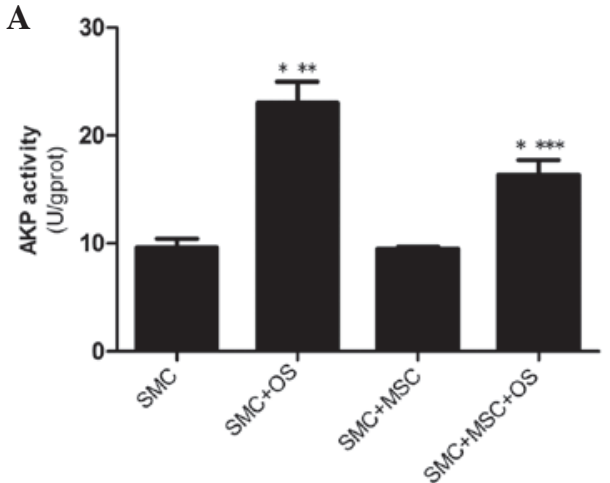

B

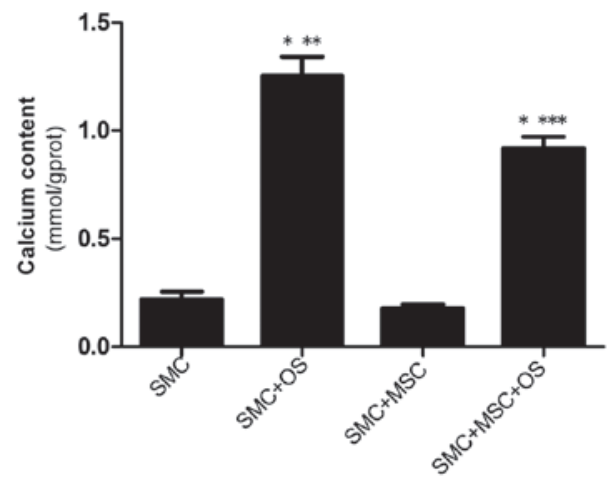

Figure 3. AKP activity and calcium content of VSMCs. VSMCs were indirectly co-cultured in the presence or absence of MSCs at a ratio of $1: 1$ for 14 days, and further tests were conducted on the VSMCs in the four groups. (A) AKP activity and (B) calcium content were significantly increased in the VSMCs treated with OS (the groups of SMC + OS and SMC + MSC + OS), and reached the highest level in the SMC + OS group . Furthermore, AKP activity and calcium content were significantly decreased in the SMC + MSC + OS group when compared with the SMC + OS group. " $\mathrm{P}<0.001$ vs. SMC group; ${ }^{* * *} \mathrm{P}<0.001 \mathrm{vs}$. other groups; ${ }^{* * *} \mathrm{P}<0.001$ vs. SMC + OS group. SMC, smooth muscle cell; VSMC, vascular SMC; MSC, mesenchymal stem cell; OS, osteogenic medium. 
A

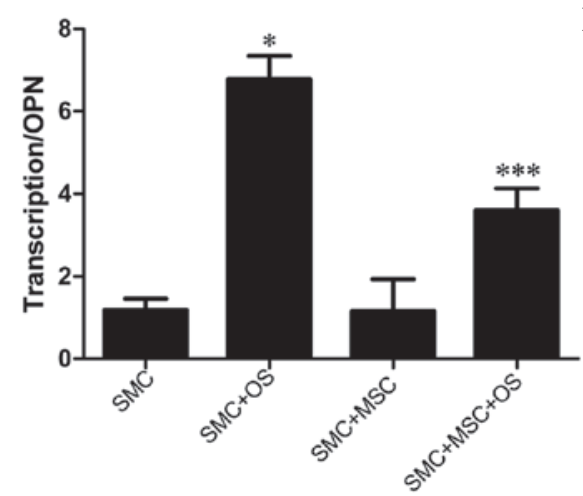

B

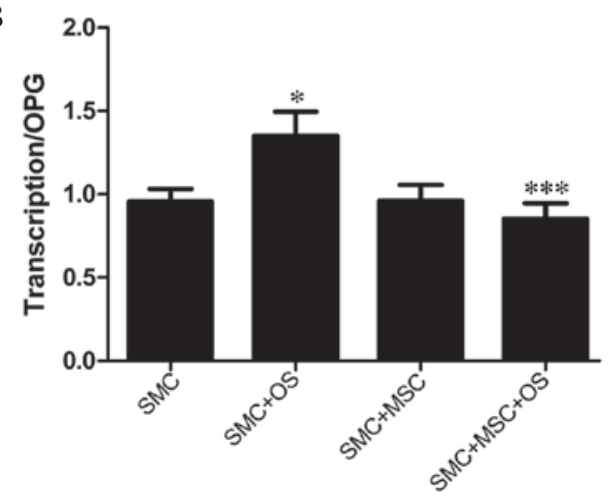

Figure 4. OPG and OPN mRNA expression. VSMCs were indirectly co-cultured in the presence or absence of MSCs at a ratio of 1:1 for 14 days, and further tests were conducted on the VSMCs in the four groups. The mRNA levels of (A) OPN and (B) OPG were significantly increased in the SMC + OS group compared with the SMC group. However, the mRNA levels were significantly reduced in the SMC + MSC + OS group when compared with the SMC + OS group. "P<0.01 vs. SMC group; ${ }^{* * * *} \mathrm{P}<0.01$ vs. SMC + OS group. OPG, osteoprotegerin; OPN, osteopontin; SMC, smooth muscle cell; VSMC, vascular SMC; OS, osteogenic medium; mRNA, messenger RNA.

\section{Discussion}

Previous studies have reported that MSCs may be cell therapy candidates for vascular regeneration and angiogenesis due to their capacity to directly migrate to sites of injury, differentiate into residential cell lineages, and secrete biochemical factors for immunomodulation and paracrine action $(7-9,12)$. The current study analyzes the anti-inflammatory, immunomodulatory and paracrine properties of MSCs on the process of VC. In order to eliminate direct mechanical stimulation, a cell-cell indirect co-culture system was constructed in vitro. Results from the present study indicate that indirect contact with MSCs inhibits VC. Furthermore, the expression levels of OPG and OPN mRNA were reduced and the Wnt signaling pathways involved were downregulated during VC.

The present study demonstrates that VSMCs trans-differentiate into osteoblast-like cells following OS induction, and promote the development of $\mathrm{VC}$, which was indicated by von Kossa staining, AKP activity and calcium content. However, the severity of calcification was controlled by indirect contact with MSCs. It is now widely accepted that MSCs isolated from bone marrow possess marked therapeutic potential for tissue repair and wound healing via cell transplantation or indirect mechanical stimulation (22-25). However, there is a limited number of studies that focus on the effects of MSCs in the treatment of VC. A recent study (26) demonstrated that MSCs are involved in $\mathrm{VC}$ as they are directed to the vascular lesions where they undergo osteogenic differentiation. Kramann et al (27) implanted bone marrow-derived MSCs intraperitoneally into two rat models of CKD with VC and demonstrated that MSCs underwent osteogenic differentiation, which contributed to VC. Wang et al (28) demonstrated that transforming growth factor- $\beta$ released from injured aortas recruited MSCs into the aortic lesions, and contributed to the development of $\mathrm{VC}$ in a low-density lipoprotein receptor-deficient mouse model that was fed a high-fat Western diet. A previous study demonstrated that MSCs trans-differentiate into osteoblast-like cells depending on the number of MSCs in direct contact with calcified VSMCs (11). Data from the above-mentioned studies indicate that MSCs promote the development of VC, when MSCs are recruited into injured lesions, via an underlying osteogenic differentiation mechanism. However, the results presented here demonstrate that MSCs exhibit therapeutic potential for treatment of $\mathrm{VC}$ via reducing AKP activity and calcium content in VSMCs. It is possible that the cell-cell indirect co-culture system may determine the effects of MSCs in VC. These results indicate that MSC-induced paracrine and immunomodulatory effects are pivotal underlying mechanisms for VC therapeutic strategies.

Bone-associated biomarkers in VSMCs, such as OPG and OPN, can be secreted by VSMCs that undergo osteogenic differentiation $(3,4)$. The present study evaluated the mRNA expression levels of these biomarkers. OPG is a soluble member of the tumor necrosis factor receptor family (5), and previous studies have demonstrated that increased OPG levels are associated with $\mathrm{VC}$ and mortality in CKD patients $(29,30)$. OPN is a glycoprotein, which is expressed in calcified vessels $(3,4,6)$ and clinical trials have indicated that OPN is an effective prognostic biomarker of coronary calcification (6). Furthermore, OPG and OPN levels are correlated with the severity of vascular mineralization $(6,29)$. In the current study, the expression levels of OPG and OPN mRNA were observed to be significantly increased in the SMC + OS group compared with the SMC group $(\mathrm{P}<0.01)$. By contrast, following indirect interaction with MSCs, the levels of OPG and OPN were significantly reduced $(\mathrm{P}<0.01)$, indicating that the extent of calcification may be increased by an underlying mechanism promoting VSMC osteogenic differentiation, and modulating OPG and OPN mRNA expression levels. Indirect contact with MSCs may prevent calcification with MSC-induced paracrine and immunomodulatory effects, as well as decreased expression levels of OPG and OPN mRNA.

The effects of MSCs on the expression of Wnt-family members, which are known to modulate the osteogenic differentiation, were also evaluated. Numerous studies have demonstrated that the Wnt signaling pathway is key in the differentiation of osteoblasts $(15,16,31)$. Canonical and noncanonical signaling pathways interact and crosstalk by binding to unrelated receptors or co-receptors $(15,16)$. It is well known that the $\beta$-catenin-dependent signaling pathway regulates osteogenic transdifferentiation and promotes vascular calcification $(15,16,32)$. By contrast, there is limited evidence that 
A

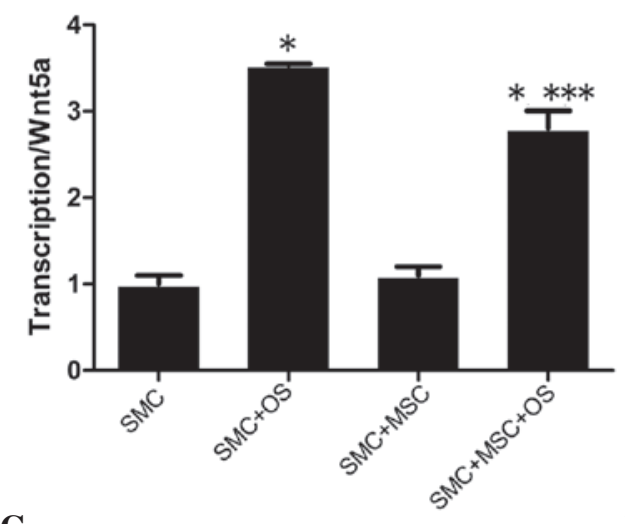

C

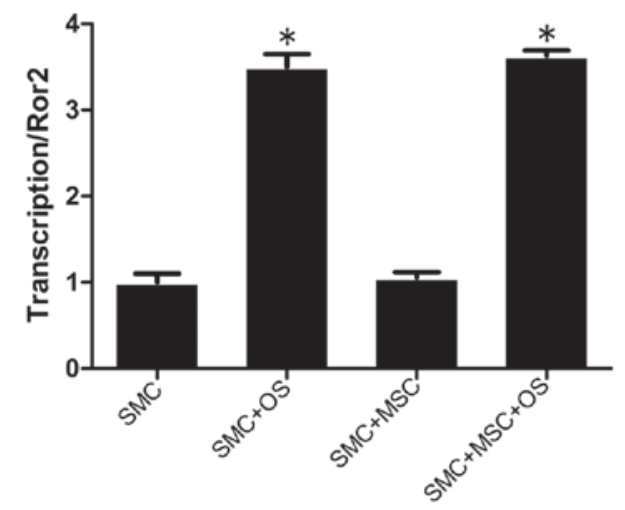

$\mathbf{E}$

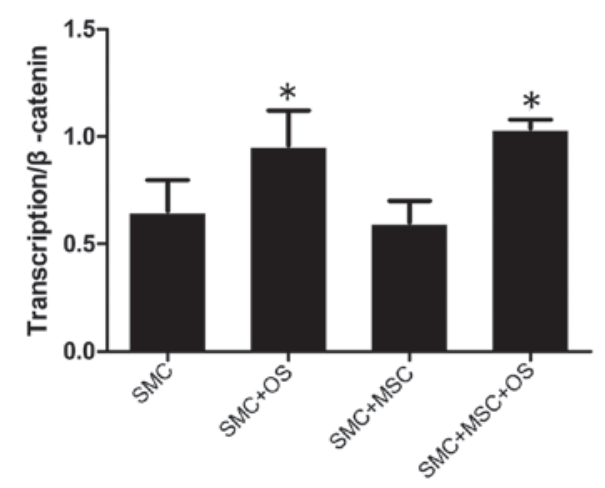

B
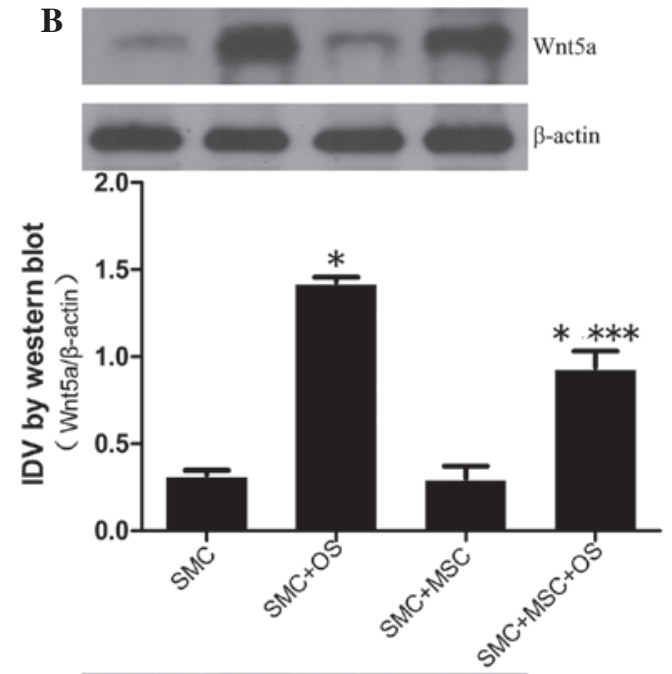

D
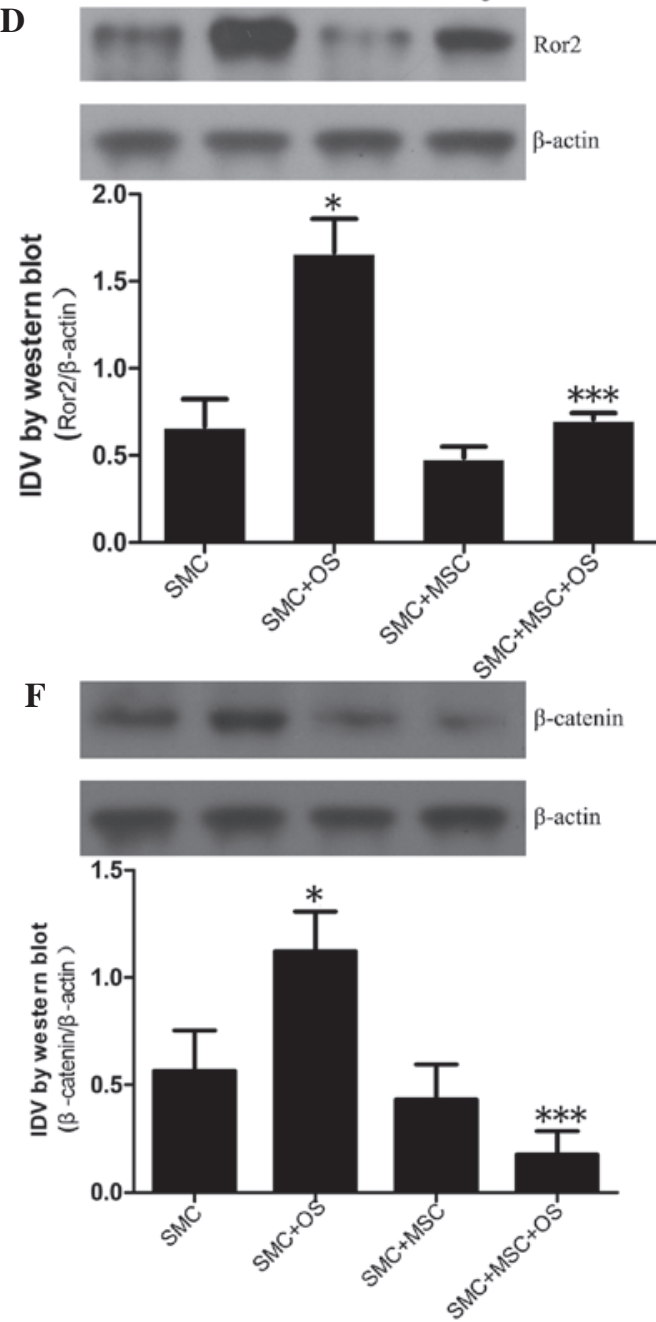

Figure 5. Wnt5a, Ror2, $\beta$-catenin expression in VSMCs. VSMCs were indirectly co-cultured in the presence of absence of MSCs at a ratio of $1: 1$ for 14 days, and further tests were conducted on the VSMCs in the four groups. The mRNA and protein expression levels of (A and B) Wnt5a, (C and D) Ror2 and $(\mathrm{E}$ and F) $\beta$-catenin were measured by RT-qPCR and western blot analysis. The mRNA expression levels of Wnt5a, Ror 2 and $\beta$-catenin were significantly increased in the groups of SMC + OS and SMC + MSC + OS when compared with the SMC group. No difference in Ror2 and $\beta$-catenin mRNA expression levels were detected between SMC + OS and SMC + MSC + OS. Compared with the SMC group, the protein levels of Wnt5a, Ror 2 and $\beta$-catenin were markedly increased in the SMC + OS group, but reduced in the SMC + MSC + OS group. $\mathrm{P}<0.001$ vs. SMC group; ${ }^{* * *} \mathrm{P}<0.01$ vs. SMC + OS group. SMC, smooth muscle cell; VSMC, vascular SMC; OS, osteogenic medum; mRNA, messenger RNA; IDV, integrated density value.

indicates the effects of noncanonical Wnt5a/Ror2 signaling in the process of VC.Xin et al (11) demonstrated that Wnt5a protein expression was associated with the severity of calcification and Ror2 mRNA expression levels were decreased when cells underwent osteogenic differentiation. However, other previous studies indicate that the activation of Wnt5a and Ror2 signals 
is associated with osteogenic differentiation. Bolzoni et al (33) demonstrated that the osteogenic differentiation of human MSCs (hMSCs) increased the expression level of Ror2 and that activation of the noncanonical Wnt5a signaling pathway also increases hMSC osteogenic differentiation. Huh et al (34) reported that arginine promotes osteogenesis in hMSCs, and increases the expression of Wnt5a via Wnt and nuclear factor of activated T-cells signaling. Liu et al (17) and Billiard et al (18) indicated that Ror2 is a modulator of osteogenesis and that increased levels of Ror 2 in hMSCs may promote formation of mineralization in the extracellular matrix.

In the present study, canonical and noncanonical signaling pathways were demonstrated to be involved in the process of VC. The expression levels of $\beta$-catenin, Wnt5a and Ror 2 are undetectable in normal VSMCs, but increase as VSMCs differentiate into the osteogenic phenotype $(11,32,33)$. The present study demonstrated that the Wnt5a/Ror2 signaling pathway inhibits Wnt/ $\beta$-catenin signaling in vitro. However, a previous study indicated a dual signaling capacity for Wnt5a in regulating Wnt/ $\beta$-catenin signaling (19). Wnt5a either inhibits or activates the Wnt $/ \beta$-catenin signaling pathway depending on its expression level and/or the receptors it is bound to. In addition, previous studies have demonstrated that Ror2 is able to bind to Wnt5a, as well as to canonical Wnt signals and mediate the action of $\beta$-catenin $(18,35)$. To the best of our knowledge, there is no direct evidence that demonstrates the underlying mechanisms of crosstalk between Wnt5a, Ror 2 and $\beta$-catenin; however, Ror2 is notably associated with VC. In addition, the current study demonstrates that MSCs have the capacity to downregulate Wnt5a, Ror2, and $\beta$-catenin expression in vitro by increasing their immunomodulatory and paracrine capacities. Thus, during the VC phase, MSCs produce factors that contribute to the prevention of the process of $\mathrm{VC}$ and downregulate canonical and noncanonical Wnt ligands.

In conclusion, the results of the present study demonstrate that VSMCs trans-differentiate into osteoblast-like cells by promoting the expression of Wnt5a, Ror2 and $\beta$-catenin. In addition, the immunomodulatory and paracrine capacities of MSCs may be associated with the prevention of VSMC osteogenic differentiation and the inhibition of VC via modulating bone-associated biomarkers and downregulating Wnt signaling pathways. This is notable for the development of stem cell-based therapeutic strategies for VC. Further studies are required that focus on determining which local mechanisms or biochemical factors maximize the inhibition of VC by MSCs. Furthermore, the crosstalk between Wnt5a, $\beta$-catenin, and Ror2 signals and the key molecular mechanisms that regulate them, remain to be elucidated.

\section{References}

1. Wu M, Rementer C and Giachelli CM: Vascular calcification: An update on mechanisms and challenges in treatment. Calcif Tissue Int 93: 365-373, 2013.

2. London GM: Mechanisms of arterial calcifications and consequences for cardiovascular function. Kidney Int Suppl (2011) 3: 442-445, 2013

3. Evrard S, Delanaye P, Kamel S, Cristol JP and Cavalier E; SFBC/SN joined working group on vascular calcifications: Vascular calcification: From pathophysiology to biomarkers. Clin Chim Acta 438 401-414, 2015.

4. McCarty MF and DiNicolantonio JJ: The molecular biology and pathophysiology of vascular calcification. Postgrad Med 126: 54-64, 2014.
5. El Hadj Othmane T, Speer G, Fekete B, Szabó T, Egresits J, Fodor E, Kiss I, Nemcsik J, Szabó A, Németh Z, et al: Osteoprotegerin: Regulator, protector and marker. Orv Hetil 149: 1971-1980, 2008 (In Hungarian)

6. Mohamadpour AH, Abdolrahmani L, Mirzaei H, Sahebkar A, Moohebati M, Ghorbani M,Ferns GA and Ghayour-Mobarhan M: Serum osteopontin concentrations in relation to coronary artery disease. Arch Med Res 46: 112-117, 2015.

7. Murphy MB, Moncivais K and Caplan AI: Mesenchymal stem cells: Environmentally responsive therapeutics for regenerative medicine. Exp Mol Med 45: e54, 2013.

8. Yagi H, Soto-Gutierrez A, Parekkadan B, Kitagawa Y, Tompkins RG, Kobayashi N and Yarmush ML: Mesenchymal stem cells: Mechanisms of immunomodulation and homing. Cell Transplant 19: 667-679, 2010.

9. Wang YK and Chen CS: Cell adhesion and mechanical stimulation in the regulation of mesenchymal stem cell differentiation. J Cell Mol Med 17: 823-832, 2013.

10. Chou SH, Lin SZ, Kuo WW, Pai P, Lin JY, Lai CH, Kuo CH, Lin KH, Tsai FJ and Huang CY: Mesenchymal stem cell insights: Prospects in cardiovascular therapy. Cell Transplant 23: 513-529, 2014.

11. Xin H, Xin F, Zhou S and Guan S: The Wnt5a/Ror2 pathway is associated with determination of the differentiation fate of bone marrow mesenchymal stem cells in vascular calcification. Int $\mathrm{J}$ Mol Med 31: 583-588, 2013.

12. Watt SM, Gullo F, van der Garde M, Markeson D, Camicia R, Khoo CP and Zwaginga JJ: The angiogenic properties of mesenchymal stem/stromal cells and their therapeutic potential. $\mathrm{Br}$ Med Bull 108: 25-53, 2013.

13. Zhang L and Xu Q: Stem/Progenitor cells in vascular regeneration. Arterioscler Thromb Vasc Biol 34: 1114-1119, 2014.

14. Kim SW, Houge M, Brown M, Davis ME and Yoon YS: Cultured human bone marrow-derived $\mathrm{CD} 31\left(^{+}\right)$cells are effective for cardiac and vascular repair through enhanced angiogenic, adhesion, and anti-inflammatory effects. J Am Coll Cardiol 64: 1681-1694, 2014

15. Marinou K, Christodoulides C, Antoniades C and Koutsilieris M: Wnt signaling in cardiovascular physiology. Trends Endocrinol Metab 23: 628-636, 2012.

16. Mill C and George SJ: Wnt signalling in smooth muscle cells and its role in cardiovascular disorders. Cardiovasc Res 95: 233-40, 2012.

17. Liu Y, Bodine PV and Billiard J: Ror2, a novel modulator of osteogenesis. J Musculoskelet Neuronal Interact 7: 323-324, 2007.

18. Billiard J, Way DS, Seestaller-Wehr LM, Moran RA, Mangine A and Bodine PV: The orphan receptor tyrosine kinase Ror2 modulates canonical Wnt signaling in osteoblastic cells. Mol Endocrinol 19: 90-101,2005.

19. van Amerongen R, Fuerer C, Mizutani M and Nusse R: Wnt5a can both activate and repress Wnt/ $\beta$-catenin signaling during mouse embryonic development. Dev Biol 369: 101-114, 2012.

20. Maeda K, Kobayashi Y, Udagawa N, Uehara S, Ishihara A, Mizoguchi T, Kikuchi Y, Takada I, Kato S, Kani S, et al: Wnt5a-Ror2 signaling between osteoblast-lineage cells and osteoclast precursors enhances osteoclastogenesis. Nat Med 18: 405-412, 2012.

21. Li X, Zhang Y and Qi G: Evaluation of isolation methods and culture conditions for rat bone marrow mesenchymal stem cells. Cytotechnology 65: 323-334, 2013.

22. Yan J, Tie G, Xu TY, Cecchini K and Messina LM: Mesenchymal stem cells as a treatment for peripheral arterial disease: Current status and potential impact of type II diabetes on their therapeutic efficacy. Stem Cell Rev 9: 360-372, 2013.

23. Hsieh JY, Wang HW, Chang SJ, Liao KH, Lee IH, Lin WS, Wu CH, Lin WY and Cheng SM: Mesenchymal stem cells from human umbilical cord express preferentially secreted factors related to neuroprotection, neurogenesis, and angiogenesis. PLoS One 8: e72604, 2013

24. King A, Balaji S, Keswani SG and Crombleholme TM: The role of stem cells in wound angiogenesis. Adv Wound Care (New Rochelle) 3: 614-625, 2014

25. Burlacu A, Grigorescu G, Rosca AM, Preda MB and Simionescu M: Factors secreted by mesenchymal stem cells and endothelial progenitor cells have complementary effects on angiogenesis in vitro. Stem Cells Dev 22: 643-653, 2013.

26. Pal SN and Golledge J: Osteo-progenitors in vascular calcification: A circulating cell theory. J Atheroscler Thromb 18: $551-559,2011$. 
27. Kramann R, Kunter U, Brandenburg VM, Leisten I, Ehling J, Klinkhammer BM, Knüchel R, Floege J and Schneider RK: Osteogenesis of heterotopically transplanted mesenchymal stromal cells in rat models of chronic kidney disease. J Bone Miner Res 28: 2523-2534, 2013.

28. Wang W, Li C, Pang L, Shi C, Guo F, Chen A, Cao X and Wan M: Mesenchymal stem cells recruited by active TGF $\beta$ contribute to osteogenic vascular calcification. Stem Cells Dev 23: 1392-1404, 2014.

29. Montañez-Barragán A, Gómez-Barrera I, Sanchez-Niño MD, Ucero AC, González-Espinoza L and Ortiz A: Osteoprotegerin and kidney disease. J Nephrol, 2014 (Epub ahead of print).

30. Morena M, Dupuy AM, Jaussent I, Vernhet H, Gahide G, Klouche K, Bargnoux AS, Delcourt C, Canaud B and Cristol JP: A cut-off value of plasma osteoprotegerin level may predict the presence of coronary artery calcifications in chronic kidney disease patients. Nephrol Dial Transplant 24: 3389-3397, 2009.

31. Guan S, Wang Z, Xin F and Xin H: Wnt5a is associated with the differentiation of bone marrow mesenchymal stem cells in vascular calcification by connecting with different receptors. Mol Med Rep 10: 1985-1991, 2014.
32. Montes de Oca A, Guerrero F, Martinez-Moreno JM, Madueño JA, Herencia C, Peralta A, Almaden Y, Lopez I, Aguilera-Tejero E, Gundlach K, et al: Magnesium inhibits Wnt/ $\beta$-catenin activity and reverses the osteogenic transformation of vascular smooth muscle cells. PLoS One 9: e89525, 2014.

33. Bolzoni M, Donofrio G, Storti P, Guasco D, Toscani D, Lazzaretti M, Bonomini S, Agnelli L, Capocefalo A, Dalla Palma B, et al: Myeloma cells inhibit non-canonical wnt co-receptor ror2 expression in human bone marrow osteoprogenitor cells: Effect of wnt5a/ror2 pathway activation on the osteogenic differentiation impairment induced by myeloma cells. Leukemia 27: 451-463, 2013.

34. Huh JE, Choi JY, Shin YO, Park DS, Kang JW, Nam D, Choi DY and Lee JD: Arginine enhances osteoblastogenesis and inhibits adipogenesis through the regulation of Wnt and NFATc signaling in human mesenchymal stem cells. Int J Mol Sci 15: 13010-13029, 2014.

35. Cai SX, Liu AR, He HL, Chen QH, Yang Y, Guo FM, Huang YZ, Liu L and Qiu HB: Stable genetic alterations of $\beta$-catenin and ROR 2 regulate the Wnt pathway, affect the fate of MSCs. J Cell Physiol 229: 791-800, 2014. 